\title{
Plasma Generation and Plume Expansion for a Transmission-Mode Micro-Laser Ablation Plasma Thruster
}

\author{
Michael Keidar and Iain D. Boyd \\ Department of Aerospace Engineering, University of Michigan, Ann Arbor MI 48109 \\ James Luke \\ University of New Mexico, Albuquerque, New Maxico 87106
}

Claude Phipps

Photonic Associates, Santa Fe, New Mexico 87505

\begin{abstract}
An end-to-end model is presented of the transient plume created by a micro laser-ablation plasma thruster. In this paper we describe a model of the plasma generation and expansion for a microlaser plasma thruster operated in transmission-mode (T-mode). The laser ablation and plasma formation processes are modeled using a kinetic ablation model. This procedure provides boundary conditions at the target surface for the plume model that is based on a particle computational approach. The present study considers a 2.5-8 $\mathrm{W}$ diode-based laser irradiating a poly-vinyl chloride target over a rectangular focal spot of $30 \times 120 \mu \mathrm{m}$ for a pulse length of 3-10 $m s$. The plume simulations reveal many details of the multi-component plasma expansion. The results are compared with experimentally obtained plume signatures. Generally good agreement between experimental and calculated flux profiles is found.
\end{abstract}




\section{Introduction}

There are many future space missions involving science, military, and commercial payloads that require very small thrust levels including very fine attitude control for high resolution Earth imaging and astronomy, and very fine positioning requirements of spacecraft formation flying that is at the core of many interferometry missions. At the same time, many basic components of spacecraft are being miniaturized so that micro-satellites and nano-satellites are being designed and built. To satisfy the needs of both the low-thrust missions and the small-scale spacecraft, miniaturized propulsion systems are under development.

Research is being conducted on a variety of micro-scale spacecraft propulsion systems in response to the needs outlined above. One type of approach involves the scaling down of largerscale thrusters to the smaller size. However, in most cases, this is only partially successful because the larger thrusters operate in a different physical regime at the small scale for which they are not optimized. For instance: cold-gas thrusters experience significantly larger boundary layers at the micro-scale in comparison to their large-scale counterparts; Hall thrusters require a significantly stronger magnetic field at the micro-scale. A separate approach to the design of micro-scale thrusters attempts to exploit physical mechanisms that work best at the micro-scale. Several micro-scale thruster concepts are currently under intensive development, such as microPulsed Plasma Thruster ${ }^{1}$ and Vacuum Arc Thruster ${ }^{2}$. An other important example of this type of micro-propulsion system is the micro laser-ablation plasma thruster ( $\mu$-LPT). Most notably, $\mu$ LPT 's have been developed by Phipps and Luke ${ }^{3}$ and Gonzalez and Baker'. In the present paper the $\mu$-LPT design of Ref. 3 is investigated and is described in detail in the following section. In Ref.4 a Q-switched micro-chip laser, pumped by a cw diode laser, was used to ablate an 
aluminum target generating thrust in the range of $0.3 \mathrm{nN}$ to $3 \mu \mathrm{N}$ with power consumption of about $5 \mathrm{~W}$ and pulse frequency in the range of $1 \mathrm{~Hz}$ to $10 \mathrm{kHz}$. The wide dynamic range of thrust levels provided by these devices is one of their most attractive features.

A key aspect in the development of any spacecraft propulsion system concerns the assessment of any potential adverse effects that the plume of the thruster may have on the spacecraft. In the case of the plasma plume generated during operation of a $\mu$-LPT, the main integration concerns are: (1) plume-induced contamination of the focal lens of the $\mu$-LPT itself (in the case of thruster operated in the so-called reflection (R)-mode); (2) contamination by the plume of other critical spacecraft components such as solar cells and optical instruments; and (3) erosion of critical spacecraft surfaces due to high energy impact by plume species.

Plume-induced spacecraft damage from a thruster is usually assessed through a combination of experimental and numerical modeling work. Experimental measurement can provide direct data that is relevant to plume effects on the spacecraft. For example, angular profiles of species mass fluxes indicate the potential for contamination, and angular profiles of ion current density accompanied by ion energy distribution functions indicate the potential for surface erosion. In addition, numerical modeling can also contribute in the assessment of spacecraft integration issues, but only if confidence in a given model can be established.

In the present paper we report on an initial attempt to formulate an end-to-end numerical model of a $\mu$-LPT. The model predicts the products of laser ablation and their properties. These data are then employed as boundary conditions in a detailed model of the plasma plume expansion process. In the following section, a description is first given of the particular $\mu$-LPT considered in the present study. Then, details are provided of the plasma formation and plasma expansion 
models. Next, results of the models are presented both for the laser ablation and the plasma plume expansion. Finally, some conclusions of the present study are provided.

\section{Micro Laser Plasma Thruster}

The micro-Laser-Ablation Plasma Thruster ( $\mu$-LPT) developed by Phipps and Luke ${ }^{3}$ uses a 1 to $10 \mathrm{~W}$, high-brightness diode laser irradiating various absorbing material and substrate combinations (e.g. black ink on paper, black PVC on kapton). Laser coupling coefficients on the order of $60 \mu \mathrm{N} / \mathrm{W}$ and specific impulses on the order of $500 \mathrm{sec}$ with a $1 \mathrm{~W}$ laser are achieved. One of the major advantages of the $\mu$-LPT is its large dynamic range of impulse bit that can be varied between $0.4 \mathrm{nN}$-s to $16 \mu \mathrm{N}$-s by simply increasing the laser pulse duration. In addition, selection of absorber and substrate materials allows the specific impulse and the laser characteristics to be tailored for specific mission requirements.

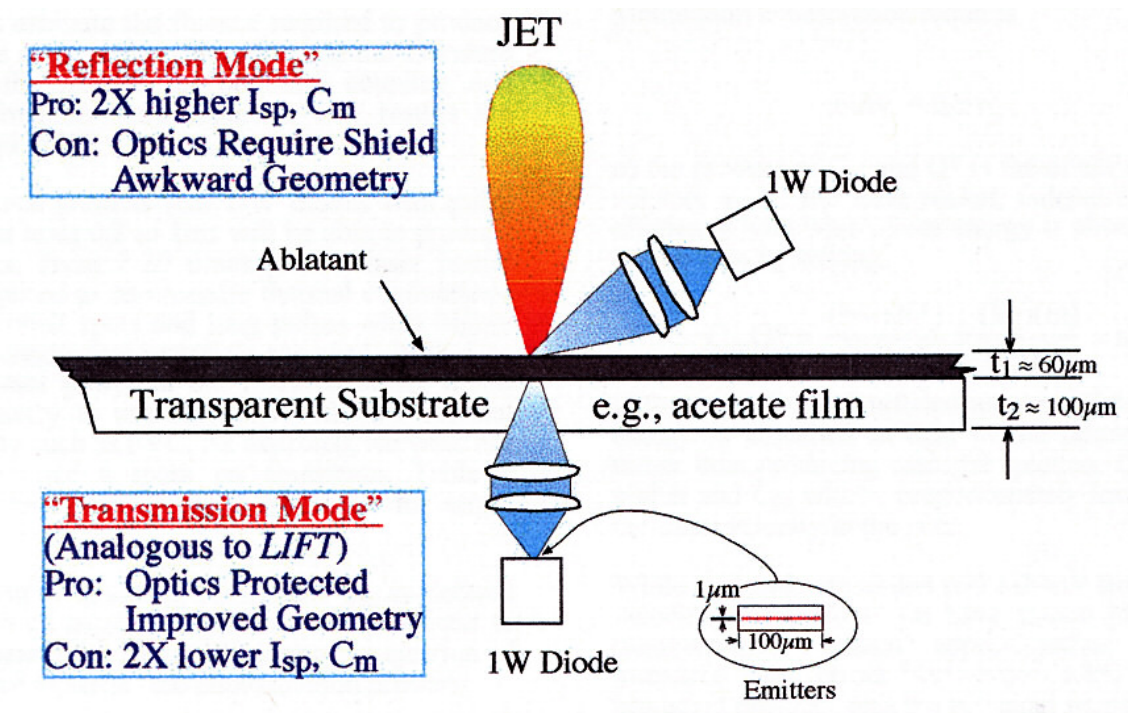

Figure 1. Schematic diagram of the $\mu-L P T$ illustrating the "reflection" $(R)$ and "transmission" $(T)$ modes. 
As illustrated in Fig. 1, the $\mu$-LPT can be operated in two different modes. In reflection mode (R), the laser is incident on the target and the ablated material "reflects" from the surface. This mode has the potential problem of leading to deposition of plume effluent on the laser optics. In transmission mode $(\mathrm{T})$, the laser passes through a transparent substrate film from the back. The substrate is coated on the other side with an absorbing material that ablates material away from the laser. This approach circumvents the problem of optics contamination found with the R mode. However, the dynamic range of impulse bit available in $\mathrm{T}$ mode is more restrictive. Coating of laser optics by plume deposition is one of the major lifetime limitations of the $\mu$-LPT in R-mode. Therefore there is a certain preference for development of T-mode operation. In this paper, we describe a model of the plasma generation and expansion for a micro-laser plasma thruster operated in transmission-mode (T-mode). Preliminary computational results for R-mode $\mu$-LPT were recently presented. ${ }^{5}$ The principle of operation of this device is shown schematically in Fig.1. A lens focuses the laser diode output on a $25 \mu \mathrm{m}$ diameter spot on the transparent side of a fuel tape. The beam heats an absorbing coating to high temperature, producing a miniature ablation jet. The material that is ablated is usually PVC or Kapton ${ }^{\mathrm{TM}}$. Typical parameters of operation are: power of 2 to $14 \mathrm{~W}$, pulse duration of 3-10 ms. The fuel tape thickness is about 185 um, composed of $125 \mathrm{um}$ of transparent backing (usually cellulose acetate) and about 60um of absorbing coating. Typically Q* (energy of laser light required to ablate one kilogram of target material) is about $2 \times 10^{7} \mathrm{~J} / \mathrm{kg}$ and the momentum coupling coefficient is about $\mathrm{C}_{\mathrm{w}}=60-100 \mu \mathrm{N} / \mathrm{W}$.

Accurate modeling of the $\mu$-LPT plume may provide predictions for the potential spacecraft integration concerns. The goal of this work is to develop models for predicting the end-to-end performance of a $\mu$-LPT, from laser-ablation of a plastic target, to the far-field of the plasma plume. In the following, we first describe a model of the formation of the plasma through laser 
ablation. Then, a description is provided of the particle model used to simulate the plasma plume expansion of the laser-ablation products.

\section{Plasma generation model}

In this section, we describe a model of the plasma layer near the evaporating surface under the effect of the laser beam. It is assumed that most of the laser absorption occurs in this layer. The plasma layer length is considered to be smaller than the laser spot diameter. The plasma layer is separated from the surface by a kinetic non-equilibrium (Knudsen) layer that has a thickness of about one mean free path as shown in Fig. 2. Here we use an approach similar to that developed for analyses of Teflon ablation under plasma effects in a pulsed plasma thruster in which the velocity at the edge of the Knudsen layer determines the evaporation rate. ${ }^{6,7}$ In general, the velocity at the edge of the Knudsen layer is actually determined by the flow state outside the Knudsen layer. ${ }^{6}$ In the case of laser-induced evaporation into vacuum, it is assumed that the ablation is free and therefore the velocity at the edge of the Knudsen layer is equal to the local sound speed. ${ }^{5}$

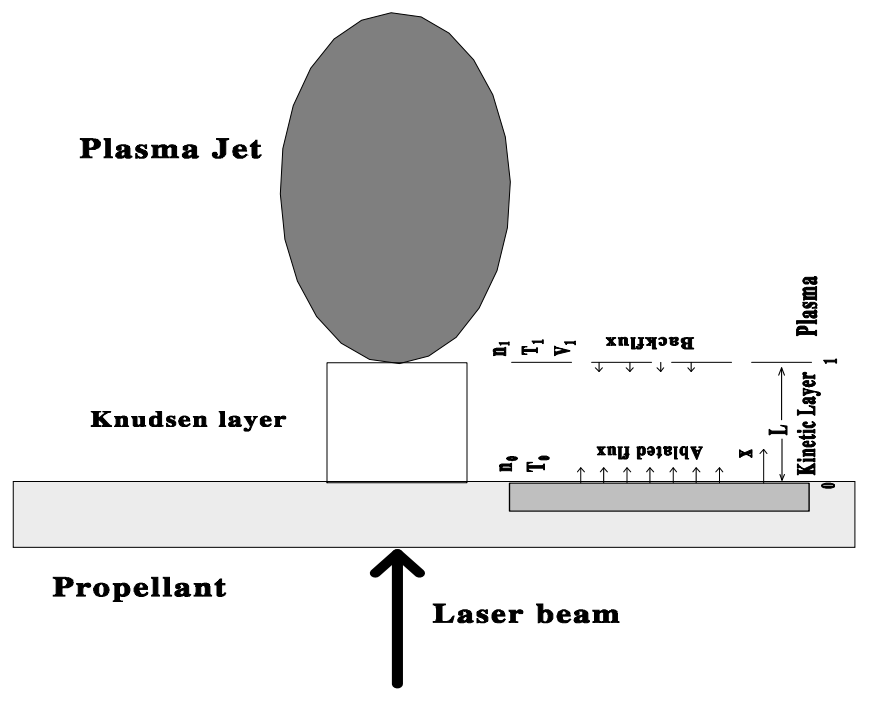

Figure 2. Schematic of the model in T-mode (not to scale). 
Solution of the Knudsen layer problem determines the boundary condition for the collisiondominated plasma. Absorption of the laser power leads to thermal evaporation, so that ejected particles obey a Maxwellian velocity distribution at the temperature of the surface $T_{\mathrm{s}}$.

In this model we assume that local thermodynamic equilibrium (LTE) is established during the laser pulse, since long pulses in the $m s$ range are considered. In this case the pulse duration is larger than the typical time for LTE formation. For instance, in a homogeneous transient plasma ${ }^{8}$, complete LTE may be obtained in $0.3 \mu$ s for a helium plasma with an electron density of $10^{24} \mathrm{~m}^{-3}$. An estimation of the characteristic times for ionization and recombination has shown that the ionization and recombination time scales for ground states of $\mathrm{C}$ and $\mathrm{H}$ are less than the typical time for the laser pulse. In the present case of $m s$ range laser pulses, the characteristic times of all collision processes are smaller than the pulse duration and therefore a quasi-steady plasma model can be used.

The ablation is modeled in the framework of the approximation ${ }^{5}$ based on a kinetic model of material evaporation into discharge plasmas ${ }^{6}$. The model couples two different layers between the surface and the plasma bulk as shown in Fig. 2: (1) a kinetic non-equilibrium layer adjacent to the surface with a thickness of about one mean free path; and (2) a collision-dominated layer with thermal and ionization non-equilibrium. The velocity at the edge of the kinetic layer $V_{l}$ can be determined from the coupling solution of the hydrodynamic layer and the quasi-neutral plasma. For known velocity and density at this interface, it is possible to calculate the ablation rate:

$\Gamma=m V_{l} N_{l}$

The system of equations is closed if the equilibrium vapor pressure can be specified that determines parameters $\left(N_{o}\right.$ and $T_{o}$ ) at the ablator surface (unfortunately equilibrium vapor pressure of the PVC is unknown and therefore we use experimentally obtained parameters to 
calculate the pressure). The solution of the Knudsen layer problem relates parameters at the boundary 1 to the parameters at the boundary 0 (Ref. 5). The full self-consistent solution of this problem can be obtained when the ablation is coupled with the plasma plume expansion. In the present work, in order to simplify the problem, we will assume that the plasma accelerates up to the sound speed near the boundary 1 . In this case the plasma density at the edge of the kinetic layer will be equal to $0.34 \cdot N_{o}$ and the temperature is $0.7 \cdot T_{o}$. The flux returned to the surface is equal to $16-18 \%$ of the ablated flux (Ref. 5). The ablated mass during the pulse can be calculated as $\Delta \mathrm{m}=\mathrm{A}_{\mathrm{S}} \Gamma \Delta \mathrm{t}$, where $\mathrm{A}_{\mathrm{S}}$ is the focal spot area and $\Delta \mathrm{t}$ is the pulse duration.

Starting from the above considerations we develope a simplified model of the plasma using the following basic assumptions: (i) the plasma is quasi-neutral and (ii) the plasma column is in Local Thermodynamic Equilibrium (LTE). The energy balance equation can be written in the form?

$\frac{3}{2} n_{e} V \partial T_{p} / \partial x=Q_{I B}-Q_{e i}-Q_{\lambda}$

where $T_{p}$ is the electron temperature in the plasma, $n_{e}$ is the electron density, $Q_{I B}=\alpha_{I B} I_{0} \exp \left(-\alpha_{I B} x\right)$ is the power density which is absorbed by electrons via the inverse bremsstrahlung effect, $Q_{e i}$ is the energy transfer from electrons to ions, and $Q_{\lambda}$ is the energy transfer due to thermal conductivity. Due to the interaction of the laser beam with the plasma, the fraction of the laser power transmitted through the plasma decreases with distance. The temperature inside the PVC ablator can be calculated from the heat transfer equation:

$\partial T / \partial t=a \not t T / \partial x^{2}$

In order to solve this equation, boundary and initial conditions must be specified ${ }^{10,11}$ : 
$\lambda \partial T / \partial x(x=0)=q(t)-\Delta H \cdot \Gamma-C_{p}\left(T_{s}-T_{o}\right) \Gamma$

$\lambda \partial T / \partial x(x=\infty)=0$

$T(t=0)=T_{o}$

where $x=0$ corresponds to the inner dielectric surface, which is in contact with a plasma, $\Delta H$ is the ablation heat, $\Gamma$ is the rate of the ablation per unit area, $T_{o}$ is the initial temperature and $q(t)$ is the laser power flux, and $T_{s}$ is the surface temperature.

Having calculated the plasma density and plasma temperature (Eqs. 1-3) one can calculate the chemical plasma composition considering Local Thermodynamic Equilibrium (LTE) in the way described previously ${ }^{8,9,12,13}$. We start our consideration from the point when we have a gas containing $\mathrm{C}$ and $\mathrm{H}$. The Saha equations for each species $(\mathrm{C}$ and $\mathrm{H})$ are supplemented by the conservation of nuclei and quasi-neutrality. The complete system of equations for chemical composition is presented in Refs.8, 9 .

At the power density level of $10^{10} \mathrm{Wm}^{-2}$ realized in the micro-laser plasma thruster ${ }^{1}$, the main mechanism of plasma absorption and therefore electron heating is the inverse bremsstrahlung. The inverse bremsstrahlung coefficient can be calculated as $[14,15]$ :

$\alpha_{I B}=1.37 \cdot 10^{-35} \lambda^{3} n_{e}^{2} T_{e}^{-1 / 2}$

where $\alpha_{I B}$ is in $\mathrm{cm}^{-1}, \lambda$ is the laser wavelength in microns, and $n_{e}$ is the electron density. 
An important parameter in laser ablation is the recoil pressure acting on the evaporating surface.

The recoil pressure can be calculated as follows ${ }^{16}$ :

$P_{r}=q_{s} / L_{v}\left(\pi k T_{s} / 2 m\right)^{0.5}$

where $\mathrm{q}_{\mathrm{s}}$ is the power density absorbed by the material. On the other hand the recoil pressure can be estimated from experimental data:

$P_{r}=C_{m} \cdot q_{s}$

where $\mathrm{C}_{\mathrm{m}}$ is the laser momentum coupling coefficient. Having calculated the ablated mass, one can estimate the parameter $\mathrm{Q}^{*}$ which is the laser energy required to ablate $1 \mathrm{~kg}$. The above system of equations allows the calculation of the plasma temperature and composition during the laser pulse. After the pulse, the plasma cools quickly by radiation and electron conductivity. The solution of the ablation model relies on several assumptions and coefficients, such as the average experimental coupling coefficient. We used these parameters because of the lack of complete data for PVC.

In this paper we consider two specific examples of a $\mu \mathrm{LPT}$ operated in T-mode for the conditions listed in Table 1 . The average laser spot size in each case is $30 \mu \mathrm{m} \times 120 \mu \mathrm{m}$.

Table 1. $\mu$ LPT conditions

\begin{tabular}{|l|l|l|}
\hline & 1 case $(01-10-30 \mathrm{a})$ & 2 case $(03-2-18 \mathrm{c})$ \\
\hline Laser power & $2.5 \mathrm{~W}$ & $8 \mathrm{~W}$ \\
\hline Background pressure & $1 \cdot 10^{-2}$ torr & $6.5 \cdot 10^{-5}$ torr \\
\hline $\begin{array}{l}\text { Experimental coupling } \\
\text { coefficient, } \mathrm{C}_{\mathrm{m}}\end{array}$ & $226 \mu \mathrm{N} / \mathrm{W}$ & $76 \mu \mathrm{N} / \mathrm{W}$ \\
\hline $\mathrm{Q}^{*}$ & $11.4 \mathrm{MJ} / \mathrm{kg}$ & $10.78 \mathrm{MJ} / \mathrm{kg}$ \\
\hline
\end{tabular}


Results are presented for calculation of the plasma generation and expansion under laser ablation of the plastic material PVC used in a micro-laser plasma thruster, PVC (poly-vinyl-chloride) contains carbon, hydrogen and chlorine. Table 2 contains a list of available fundamental properties of PVC.

Table 2. Physical properties of PVC

\begin{tabular}{|l|l|}
\hline Density & $1439 \mathrm{~kg} / \mathrm{m}^{3}$ \\
\hline Thermal conductivity & $0.15 \mathrm{~W} / \mathrm{mK}$ \\
\hline Specific heat & $1.7 \mathrm{~kJ} / \mathrm{kgK}$ \\
\hline Heat of vaporization & $0.284 \mathrm{MJ} / \mathrm{kg}$ \\
\hline
\end{tabular}

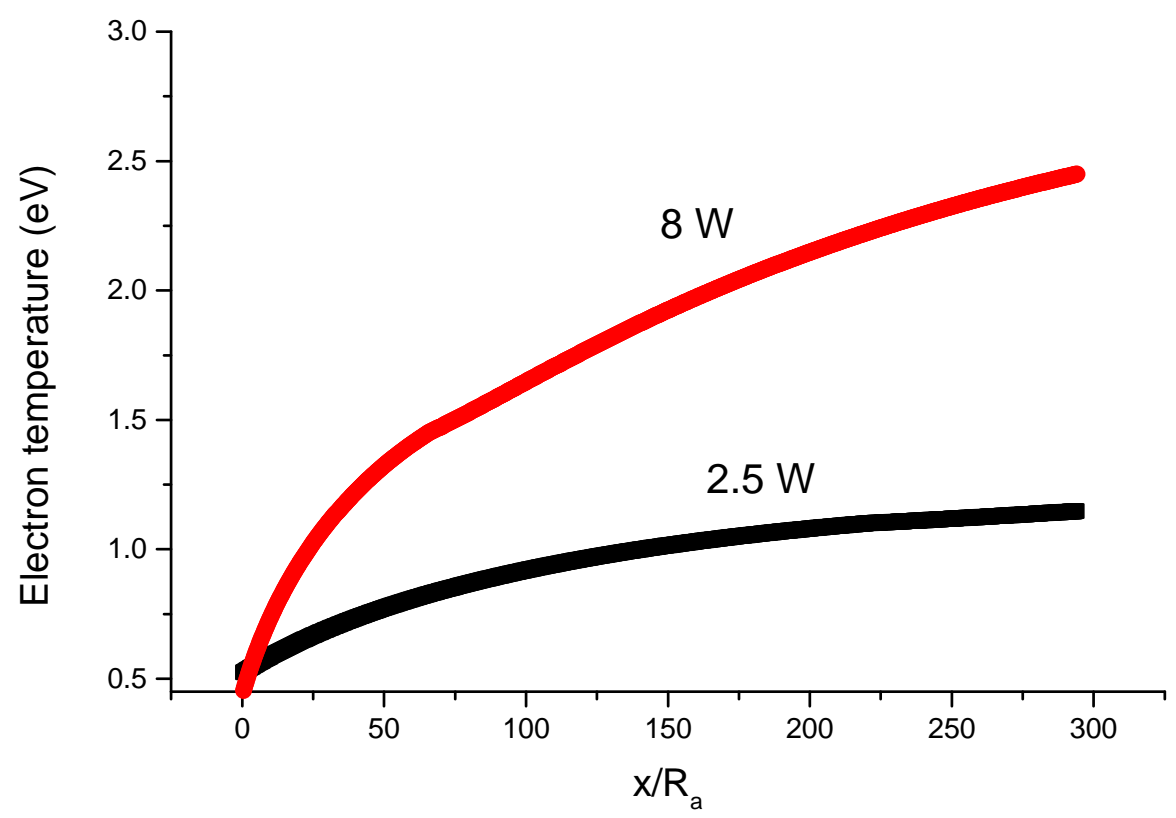

Figure 3. Electron temperature distribution along the plasma jet originated from the target surface. Parameters correspond to 2 cases presented in Table 1.

The electron temperature in the plasma generation region is shown in Fig. 3. Two cases (see Table 1) are compared. One can see that the case with higher power produces a plasma with a much higher electron temperature. Dependent on the electron temperature, one can expect that the 
plasma composition will also be different. The results of the plasma composition calculations are summarized in Table 3.

Table 3. Results of plasma generation modeling

\begin{tabular}{|l|l|l|}
\hline & Case 1 (01-10-30a) & Case 2 $(03-2-18 \mathrm{c})$ \\
\hline Laser power $(\mathrm{W})$ & 2.5 & 8 \\
\hline Background pressure (torr) & $1 \cdot 10^{-2}$ & $6.5 \cdot 10^{-5}$ \\
\hline Electron temperature $(\mathrm{eV})$ & 1.147 & 2.45 \\
\hline Electron density $\left(\mathrm{x} 10^{23} \mathrm{~m}^{-3}\right)$ & 6.836 & 30.625 \\
\hline $\mathrm{C}$ atom density $\left(\mathrm{x} 10^{23} \mathrm{~m}^{-3}\right)$ & 10.258 & 0.2 \\
\hline $\mathrm{C}$ ion density $\left(\mathrm{x} 10^{23} \mathrm{~m}^{-3}\right)$ & 3.9 & 10.227 \\
\hline $\mathrm{H}$ atom density $\left(\mathrm{x} 10^{23} \mathrm{~m}^{-3}\right)$ & 25.4 & 0.46 \\
\hline $\mathrm{H}$ ion density $\left(\mathrm{x} 10^{23} \mathrm{~m}^{-3}\right)$ & 2.929 & 20.398 \\
\hline Velocity $(\mathrm{m} / \mathrm{s})$ & 4838 & 7090.37 \\
\hline
\end{tabular}

In these calculations we assume that all particles have the same temperature and also the same center-of-mass velocity ${ }^{17}$. It can be noted from Table 3 that in the second case, the plasma is highly ionized, while in the first case the ionization degree is small. It will be shown below that the plasma composition will affect the plasma plume flowfield.

\section{Plasma Plume Expansion Model}

Models for the plumes created by laser ablation have been described previously by Itina et al. ${ }^{18}$ and by Franklin and Thareja. ${ }^{19}$ In each case, Monte Carlo methods were employed to analyze the effects of a finite back pressure on the plume expansion while the plasma was modeled as a fluid. The focus of these studies was on use of laser ablation for thin film deposition. The laser spot size was significantly larger in those studies in comparison to that for the $\mu$ LPT considered here.

In the present investigation, a hybrid fluid-particle approach is employed. The heavy-particle products of the laser ablation of PVC (neutral atoms of $\mathrm{C}$ and $\mathrm{H}$, and ions $\mathrm{C}+$ and $\mathrm{H}+$ ) are modeled as particles. Particle collisions are computed using the direct simulation Monte Carlo method (DSMC).$^{20}$ Both momentum exchange and charge exchange collisions are simulated. 
Momentum exchange cross sections follow the model of Dalgarno et al. ${ }^{21}$ and the collision dynamics follows the normal DSMC procedures as described in Ref.18. The charge exchange processes employ the cross sections proposed by Sakabe and Izawa. ${ }^{22}$ No momentum exchange is simulated during charge exchange.

Acceleration of the charged particles in self-consistent electric fields is simulated using the Particle-In-Cell method (PIC). ${ }^{23}$ The plasma potential, $\varphi$, is obtained by assuming charge neutrality to determine the electron number density from the total ion density. By further assuming the electrons are adiabatic, the electron number density, $n_{e}$, is then used in the Boltzmann relation to obtain the plasma potential:

$\varphi-\varphi^{*}=T^{*} \frac{\gamma}{\gamma-1}\left[\left(\frac{n_{e}}{n^{*}}\right)^{\gamma-1}-1\right]$

where $\varphi^{*}, T^{*}$ and $n^{*}$ are reference values and $\gamma=5 / 3$. This approach has been used successfully in our previous work on modeling the plumes of Hall thrusters. In the case of the $\mu$-LPT, the reference point for the Boltzmann relation is taken as the target surface. It is assumed that the potential here is constant.

The simulation uses a single grid for both the collision and plasma processes. Since charge neutrality is assumed, the PIC cells are not required to be of the order of the Debye length. Instead they are chosen to be small enough to resolve in a reasonable way the gradients in the potential. At the same time, the cells satisfy the DSMC requirement that their size be less than a mean free path.

The experimental facility back-pressure of the order of $10^{-5}-10^{-2}$ torr is simulated. In the simulations, this pressure is applied as a fixed background condition with which particles from the thruster can collide. The back-pressure gas is assumed to be fully composed of hydrogen atoms at a temperature of $300 \mathrm{~K}$. 


\section{Results}

For simplicity, we consider a two-component plasma consisting of carbon and hydrogen. The chlorine component is omitted due to its relatively low abundance. Two examples of a $2.5-8 \mathrm{~W}$ diode laser beam focused on a 30x120 m radius spot is considered. The pulse duration is ms, and the experimentally measured thrust-to-power (coupling coefficient) of about 70-200 $\mu \mathrm{N} / \mathrm{W}$ (see Table 1) is used in order to estimate the recoil pressure.

The particles injected into the DSMC-PIC simulation are sampled from the equilibrium velocity distribution corresponding to the temperatures and velocities mentioned in Table 3. A similar approach has been successfully validated in previous work on simulating the plumes generated in Electron Beam Physical Vapor Deposition processes. ${ }^{24}{ }^{25}$

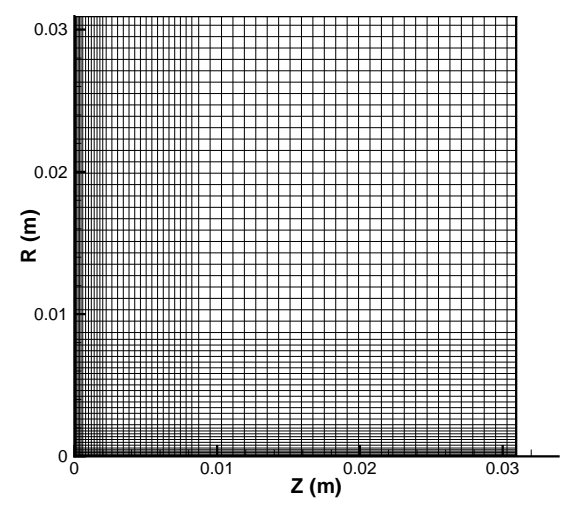

Figure 4. Computational mesh in DSMC-PIC plume simulation

The grid employed in the plume computation contains 300 by 300 non-uniform rectangular cells, and is shown in Fig. 4 (only 1 in every 5 cells is displayed). The flow domain extends to about 3 $\mathrm{cm}$ in both the axial and radial directions from the center of the ablation spot. This region is 
chosen to cover the area within which the optics of the thruster lies so as to allow assessment of potential contamination. A constant time-step of $2 \times 10^{-10} \mathrm{sec}$ is employed that is smaller than the smallest collision and plasma time scales (the inverses of the maximum collision and plasma frequencies, respectively).

A steady state is reached after 40,000 iterations and final results are obtained by averaging over a further 50,000 steps. A maximum of more than 1 million particles is employed in the simulation.
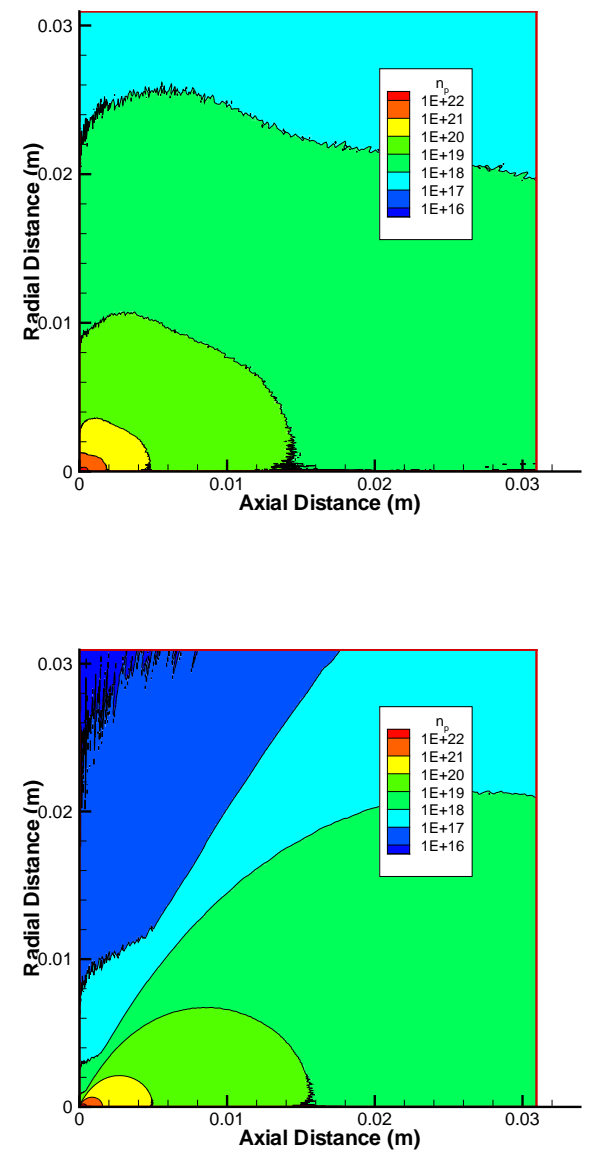

Figure 5. Contour plots of the plasma number density. Case 2 (Table 3). Upper figure: $P=6.5 \times 10^{-5}$ torr, lower figure: vacuum.

Contours of plasma densities are shown in Fig. 5. One can see that background gas pressure strongly affect the plume expansion due to CEX and momentum collisions. 
The heavy particles energy distribution at $1 \mathrm{~cm}$ and $45^{\circ}$ from the spot center is shown in Figs. 6 for higher power case 2 (Tables 1,3). One can see that carbon ions and atoms have a higher most probable energy and their distribution is broader than that for the hydrogen ions and atoms. In the case of the finite background pressure, Fig. 6b, the probability of charge exchange collisions (CEX) increases. In this case a noticeable effect of CEX collisions is obtained in which a significant population of low energy ions is created.

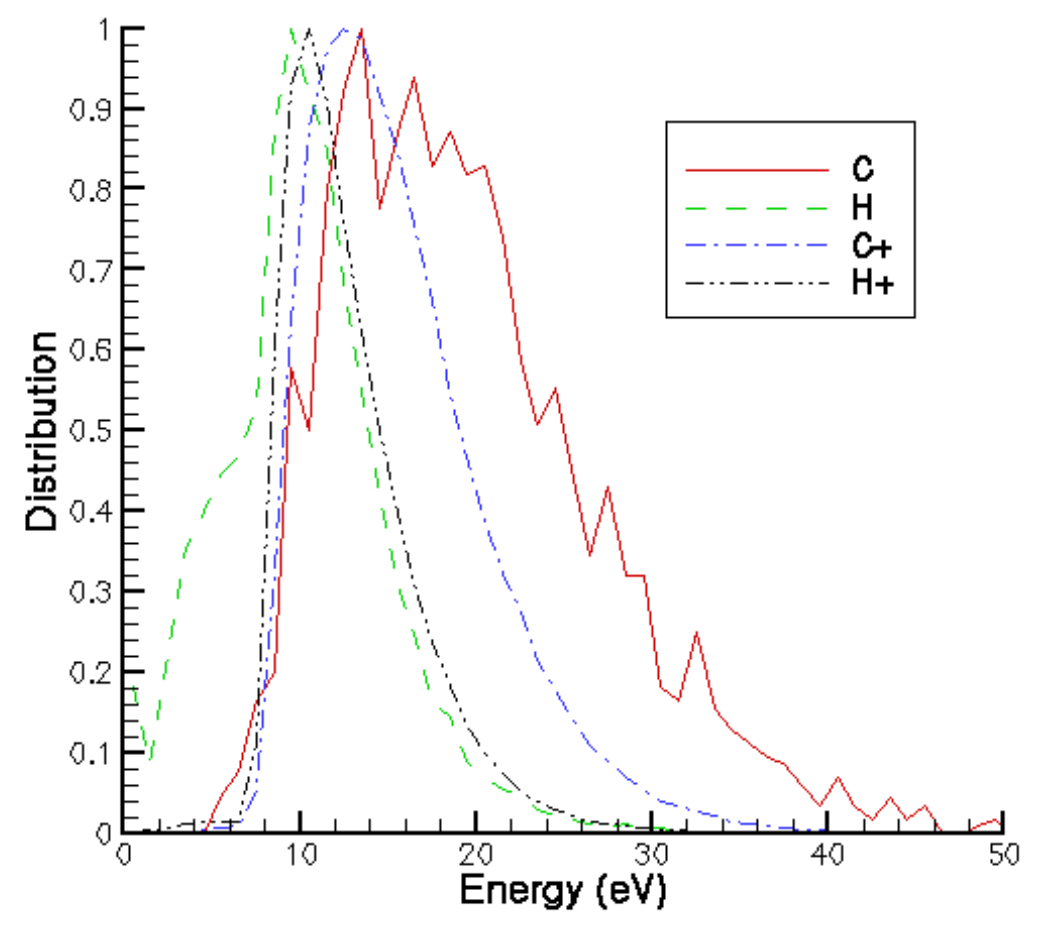

Fig. 6a. Energy distribution. Vacuum 


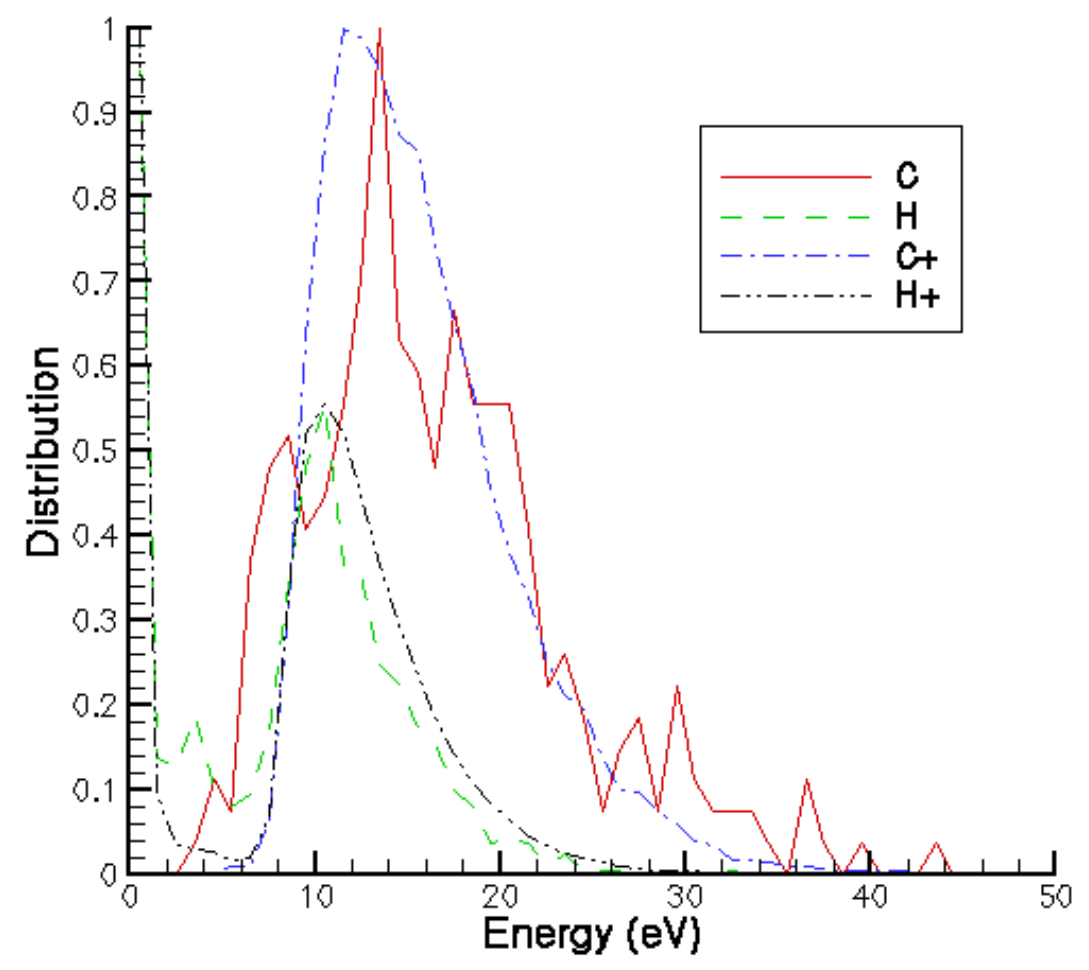

Fig. 6b. Energy distribution. $P=6.5 \times 10^{-5}$ torr

Table 4. Backflux (ratio of the backward mass flux to the outward mass flux)

\begin{tabular}{|l|l|l|}
\hline & Case 1 & Case 2 \\
\hline vacuum & 0.018 & 0.002556 \\
\hline Background pressure, $6.5 \times 10^{-5}$ & 0.0184 & 0.0107 \\
\hline $1 \times 10^{-2}$ & 0.0196 & ------ \\
\hline
\end{tabular}

The calculated backflux fraction is shown in Table 4. In all cases the level of backflux is very small (2\% at most) although there is a noticeable effect of background pressure. 


\section{Comparison with experiment}

The distribution of plume deposition on a witness plate was obtained experimentally. The schematic of the experiment is shown in Fig. 8 and plume signatures are shown in Figs. 9. The axes in this figure are labeled in degrees, with $0^{\circ}$ corresponding to the normal to the tape surface.

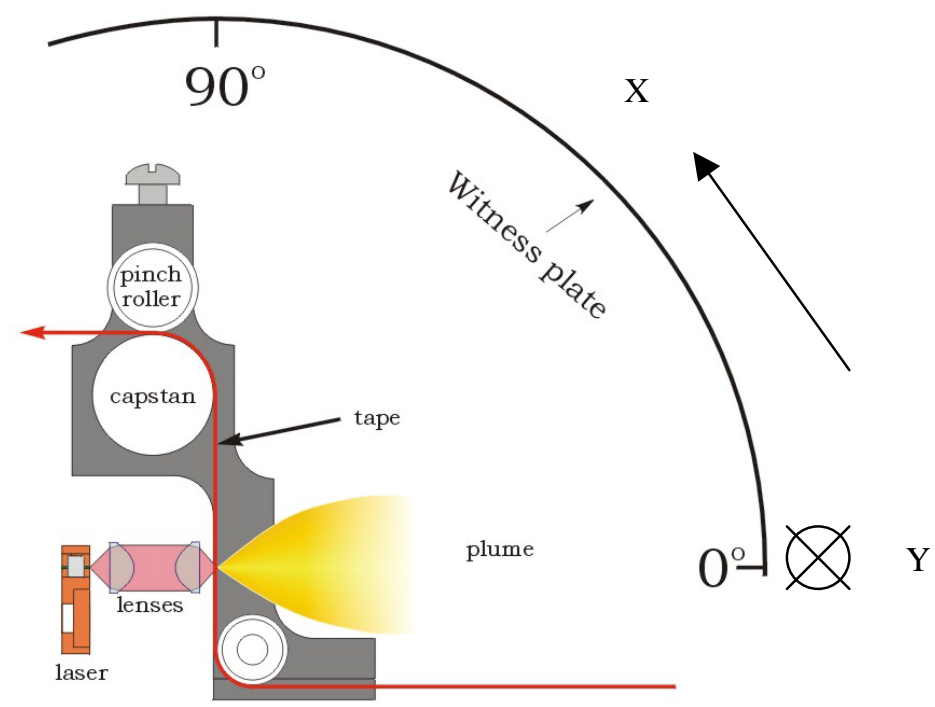

Figure 7. Schematic of the witness plate setup.

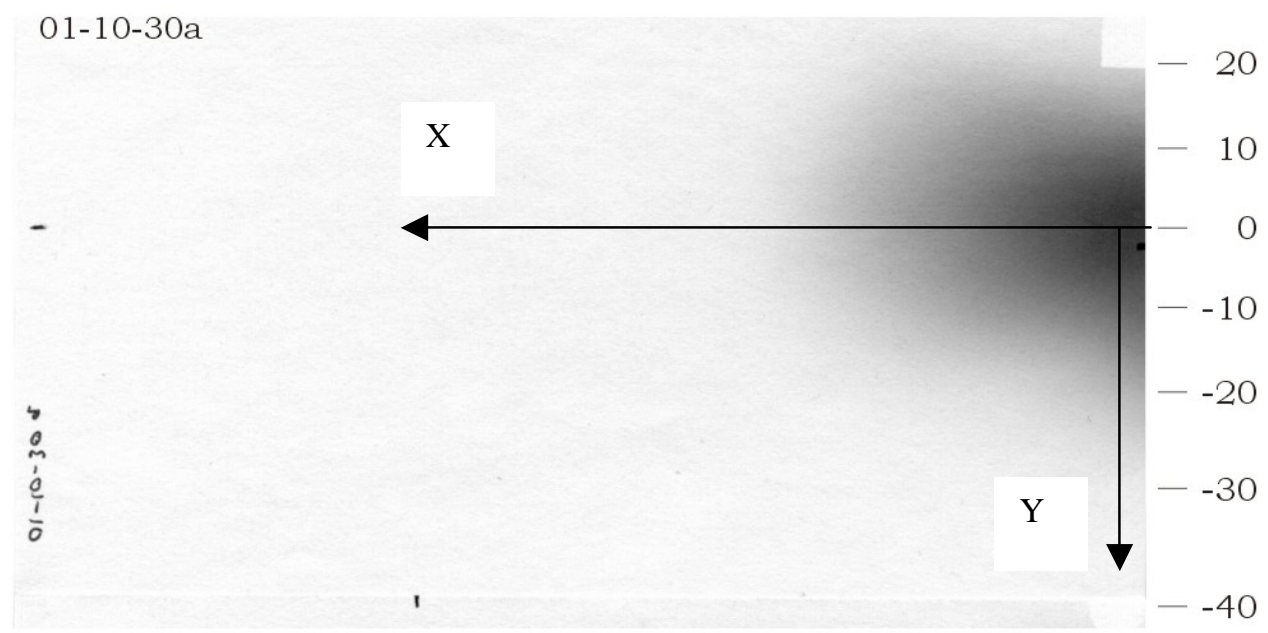

Figure $8(a)$. Plume signature. High pressure case $1 \times 10^{-2}$ torr 


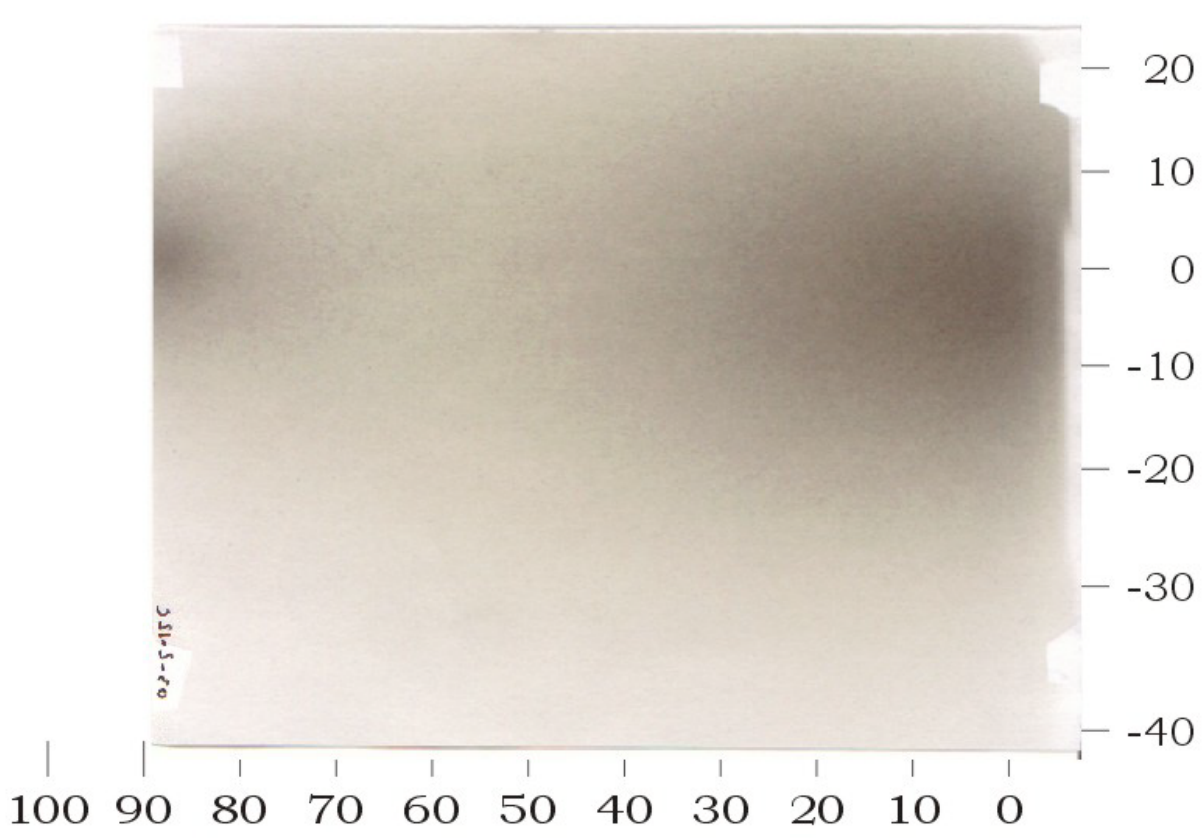

Fig. 8b. Plume signature. (b) low pressure case $6.5 \times 10^{-5}$ torr

The distance from the laser focus to the cylinder on which the witness plate is rolled is $6 \mathrm{~cm}$. The cylinder is made of metal and mounted to the vacuum chamber so that it is always in the same location. The $\mathrm{X}$-axis in the images is the distance around the circumference of the cylinder. The Y-axis is the distance along the length, and the degree marks are not equally spaced. One can see that in the low-pressure case plasma plume spreads significantly in comparison with highpressure case. The witness plate depositions are analyzed using the software Scion Image ${ }^{26}$. Using this software the density distribution is quantified (in our case in the $\mathrm{X}$ and $\mathrm{Y}$ directions across the image, as shown in Figs. 7,8a). 


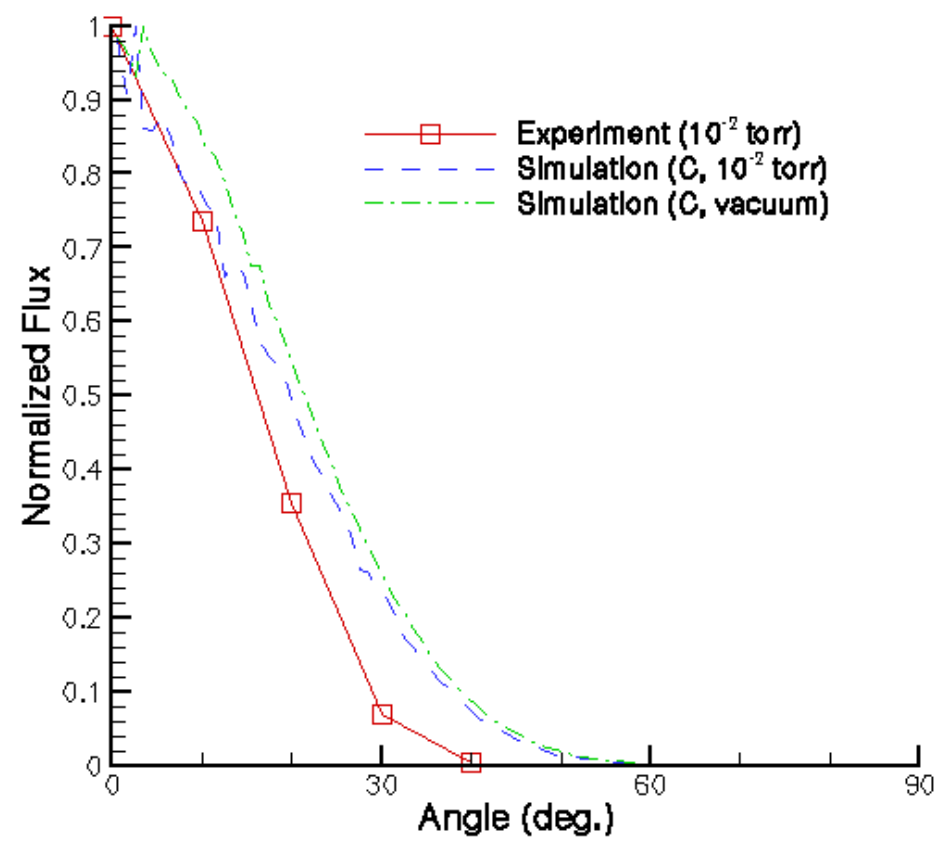

Figure 9a. Normalized flux distribution. High pressure, low power (case 1), a) X direction

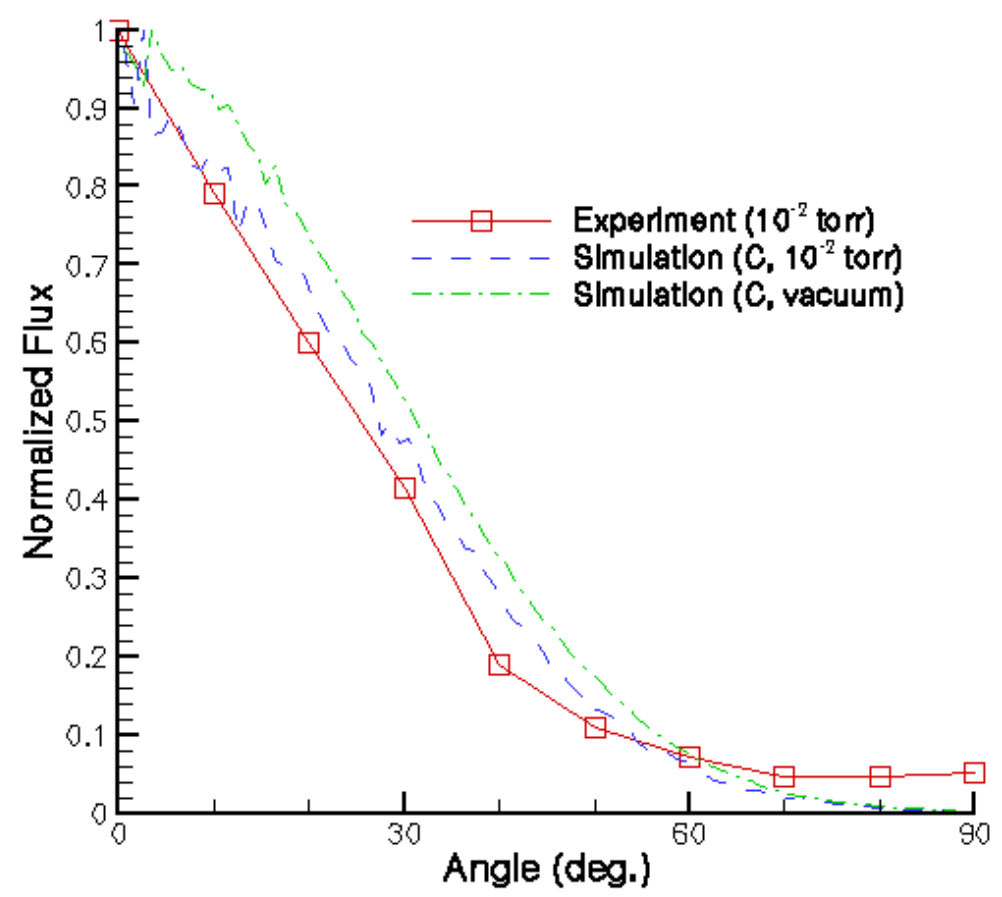

Figure 9b. Normalized flux distribution. High pressure, low power (case 1), b) Y direction 


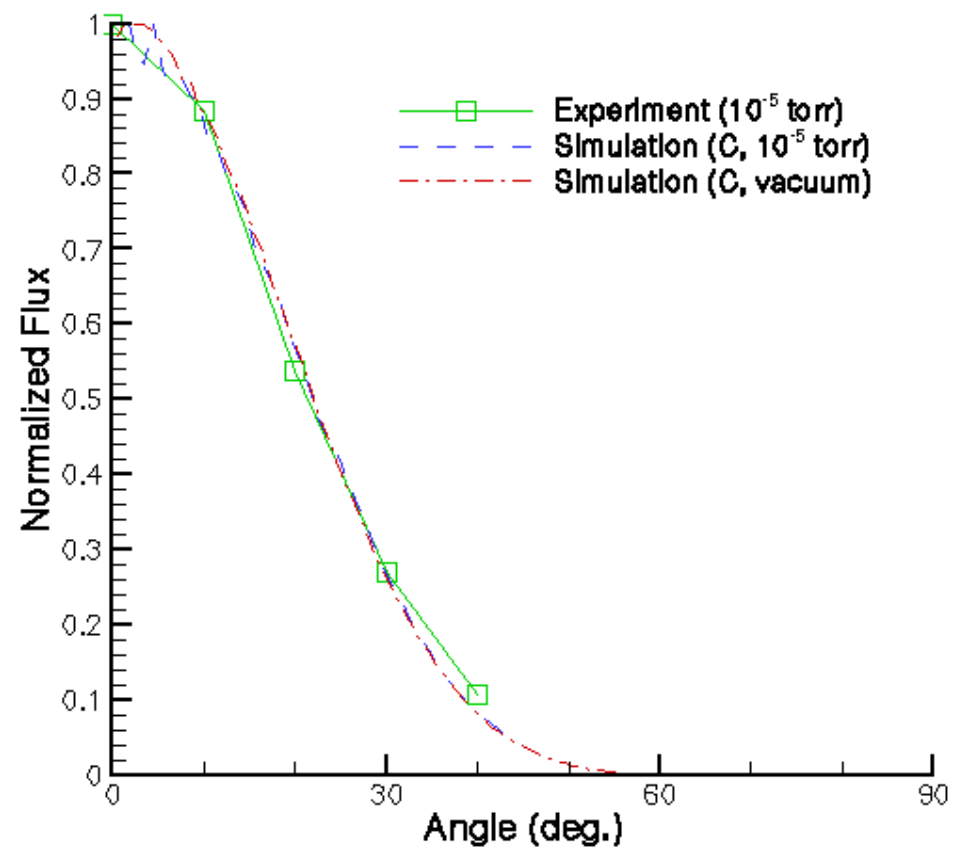

Figure 10a. Normalized flux distribution. Low pressure, high power (case 2), a) X direction

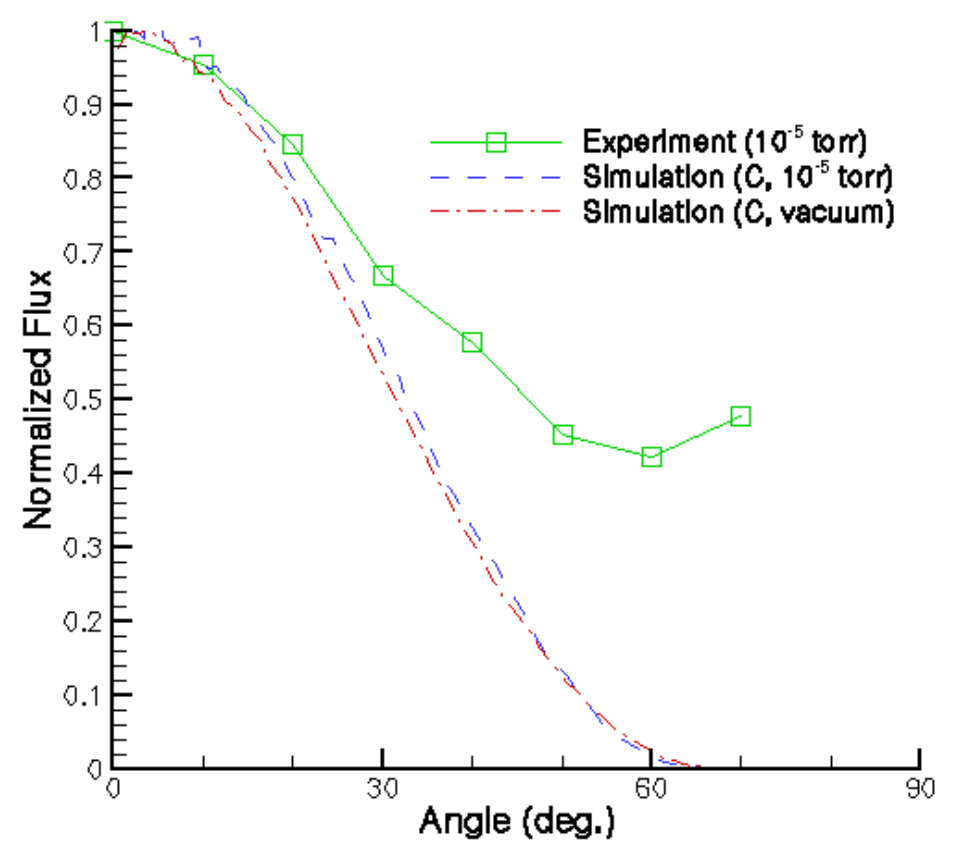

Figure 10b. Normalized flux distribution. Low pressure, high power (case 2), b) Y direction 
The normalized flux profiles are shown in Figs. 9,10 for all cases (low and high power and low and high pressure, and vacuum). It is assumed that the black deposition material on the witness plate is carbon and so the simulation results in Figs. 9,10 contain the total fluxes of carbon ions and atoms. One can see clear differences in the two deposition signatures and these are due to two effects, namely background pressure and operational conditions. The strongest effect of the background pressure can be seen in the higher pressure (case 1) case while in the low pressure case calculations in the vacuum case produces similar results. It should be noted that the experimental distribution shown in Fig. 10b has a second peak, which is not simulated. This peak is due to deflection of the exhaust plume. It happens probably due to the fact that when the laser ablates the fuel material and burns into the substrate, the edge of the trench forms a plumesteering surface. ${ }^{27}$ One can conclude that overall the comparison of the experimental results and predictions show generally good agreement in the flux profiles in both $\mathrm{X}$ and $\mathrm{Y}$ directions.

\section{Concluding remarks}

An end-to-end modeling procedure was formulated for predicting the plasma plume structure generated by a micro-laser-ablation plasma thruster operated in T-mode. The model described the laser ablation of a plastic target, the plasma generation at the target surface, and the plasma plume expansion into the far-field. The plasma formation model was based on a semi-analytical fluid approach and assumed equilibrium to calculate the plasma composition. The plasma plume was modeled using a DSMC-PIC approach to include both gas-dynamic and electro-static acceleration. The plume simulation provided details of the expansion process such as energy distribution functions and deposition profiles. Such data can be used to assess spacecraft contamination issues for the thruster. The plasma plumes were investigated experimentally using 
depositions on witness plates. These results were compared with model predictions. Generally good agreement between experimental and calculated flux profiles was found.

\section{ACKNOWLEDGMENTS}

The work at the University of Michigan was funded by the Air Force Office of Scientific Research under Grant F49620-02-1-0084 with Dr. Mitat A. Birkan as the technical monitor. This The work at Photonics Associates and University of New Mexico was supported by the U.S. Air Force under contracts F04611-02-M-0025, F49620-00-C-0005, and F49620-980C-0038

\section{References}

${ }^{1}$ G. Spanjers, D. R. Bromaghim, Capt. J. Lake, M. Dulligan, D. White, J. H. Schilling, S.S. Bushman, E. L. Antonsen, R.L. Burton, M. Keidar and I. D. Boyd, "AFRL microPPT development for small spacecraft propulsion", $28^{\text {th }}$ AIAA Joint Propulsion Conference, Indianapolis, IN, USA, July 2002, Paper AIAA-2002-3974.

${ }^{2}$ J. Schein, N.Qi, R. Binder, M. Krishnan, J. K. Ziemer, J.E. Polk and A. Anders, IEPC-01-238

${ }^{3}$ C. Phipps and J. Luke, “'Diode laser-driven microthrusters: a new departure for micropropulsion," AIAA Journal 40, pp. 310--317, 2002.

${ }^{4}$ D.A. Gonzalez and R.P. Baker, “'Microchip Laser Propulsion for Small Satellites," AIAA Paper 2001-3789, 37 ${ }^{\text {th }}$ AIAA/ASME/SAE/ASEE Joint Propulsion Conference, July 2001.

${ }^{5}$ I. D. Boyd and M. Keidar, Simulation of the plume generated by a micro laser-ablation plasma thruster, High-Power Laser Ablation IV, C.R. Phipps, Ed., Proc. SPIE, vol. 4760, 2002, p. 852 
${ }^{6}$ M. Keidar, J. Fan, I.D. Boyd and I.I. Beilis, "Vaporization of heated materials into discharge plasmas”, Journal of Applied Physics, vol. 89, No. 6, pp. 3095-3098 (2001)

${ }^{7}$ M. Keidar, I.D. Boyd and I.I. Beilis, On the model of Teflon ablation in an ablation-controlled discharge, J. Phys. D: Appl. Phys., 34, June, 2001, pp. 1675-1677.

${ }^{8}$ H. R. Griem, Plasma Spectroscopy, McGraw-Hill Book Company, New York, 1964.

${ }^{9}$ Principles of laser plasmas, Ed. G. Bekefi, Wiley, 1976, p. 527.

${ }^{10}$ M. Keidar, I.D. Boyd and I.I. Beilis, Electrical discharge in the Teflon cavity of a co-axial pulsed plasma thruster, IEEE Trans. Plasma Sci., 28, 2000, pp. 376-385.

${ }^{11}$ Keidar, I.D. Boyd and I.I. Beilis, Model of an elecrothermal pulsed plasma thruster, Journal Propulsion \& Power, 19, No. 3, 2003, pp. 424-430.

${ }^{12}$ P. Kovatya, "Thermodynamic and transport properties of ablated vapors of PTFE, alumina, perspex and PVC in the temperature range 5000-30000 K”, IEEE Trans. Plasma Sci., 12, 1984 pp. $38-42$.

${ }^{13}$ C.S. Schmahl and P.J. Turchi, , "Development of equation-of-state and transport properties for molecular plasmas in pulsed plasma thrusters. Part I: A two-temperature equation of state for Teflon”, Proc. Inter. Electr. Propul. Conf. Pp. 781-788, $1997 .$.

${ }^{14}$ Y. Zeldovich and Yu. Raiser, Physics of shock waves and high-temperature hydrodynamics phenomena, p. 259, Academic, New York, 1966.

${ }^{15}$ J.J. Chang and B.E. Warner, “'Laser-plasma interaction during visible-laser ablation of methods," Appl. Phys. Lett. 69, pp. 473--475, 1996.

${ }^{16}$ Xi Chen and H.X. Wang, Theoretical modeling of rapid surface vaporization with back pressure, J. Phys. D: Appl. Phys., 34, pp. 2637-2642, 2001

${ }^{17}$ R. Kelly and R.W. Dreyfus, Nucl. Instr. Phys. Res., B32 341, 1988 
${ }^{18}$ T.E. Itina, W. Marine, and M. Autric, Mathematical modeling of pulsed laser ablated flows, Appl. Surf. Sci., 154-155, p. 60, 2000

${ }^{19}$ S.R. Franklin and R.K. Thareja, Monte Carlo simulation of laser ablated plasma for thin film deposition, Appl. Surf. Sci., 177, p.15, 2001

${ }^{20}$ G.A. Bird, "Molecular gas dynamics and the direct simulation of gas flows" (Clarendon Press, Oxford, 1994).

21 A. Dalgarno, M.R.C. McDowell and A. Williams, The modilities of ions in unlike gases, Proc. Of Royal Soc. Of London, Vol. 250, April 1958, pp. 411-425.

${ }^{22}$ S. Sakabe and Y. Izawa, Simple formula for the cross sections of resonant charge transfer between atoms and their ions at low impact velocity, Physical Rev. A: General Physics, v. 45, No. 3, 1992, pp. 2086-2089.

${ }^{23}$ C.K. Birdsall and A.B. Langdon, Plasma Physics via Computer Simulation, Adam Hilger Press, 1991.

${ }^{24}$ J. Balakrisnan, I. D. Boyd and D.G. Braun, J. Vac. Sci Technol., A18, p. 907, 2000

${ }^{25}$ J. Fan, I.D. Boyd and C. Shelton, J. Vac. Sci. Technol., A18 p. 2937, 2000

${ }^{26}$ www.scioncorp.com

${ }^{27}$ J. R. Luke, C.R. Phipps and G.G. McDuff, laser plasma thruster continuous thrust experiment, , High Power Laser Ablation IV, C.R. Phipps, Ed., Proc. SPIE, vol. 4760, 2002, p. 843 